\title{
Utility of Bone Scan Quantitative Parameters for the Evaluation of Prostate Cancer Patients
}

\section{Nayab Mustansar*}

Nuclear Physician, AFIP (Armed Forces Institute of Pathology), Islamabad, Pakistan

\begin{abstract}
Prostate Cancer is one of the common cancers in the world. It could primarily disseminate to the bone and can lead to death. In order to address its life threatening distant metastasis it is important to diagnose it earlier for timely treatment. Bone metastasis is usually diagnosed deploying bone scan imaging. However interpretation of the bone scans is a tedious procedure for the physicians and often leads to misinterpretation either as overestimation or underestimation of the metastasis. To minimize the risk of misinterpretation, one of the accurate methods is quantitative analysis of the bone scans in order to ascertain, whether a metastatic lesion is present or not. There are several methods to-date which can be used to analyze the extent of such lesions. For example, quantitation of the bone scan using quantitation methods i.e. \%BSI (Bone scan index), \%PAB (Positive area on bone scans), EOD (extent of disease) and BLS (Bone lesion scoring). These methods are used for prognostication of survival and response to treatment on serial scans. The extent of fidelity of these all available quantitation methods is not clear when used altogether in a single baseline bone scan. Therefore, the aim of this study is to use all available bone scan quantitative parameters on a baseline bone scans and to compare them all. Moreover, an improved methodology is introduced by comparing the results with the individual methods reported in literature and with PSA levels.

141 patients with histopathologically proved prostate cancer were chosen to implement all the four quantitative parameters on individual baseline bone scans. After which, for the calculation of risk of progression or regression of disease and survival rate, 40 patients were chosen from the same dataset. A serial follow up scan was performed to calculate 2-years survival rate. The dataset was again analyzed using the same four bone scan quantitative parameters and the cut off were calculated as \%BSI: 1, \%PAB: 0.5, EOD: grade 0 \& 1, grade 2, 3 \& 4 and BLS: 5 .

It was found out that the \%PAB and \%BSI methods are good prognostic indicator in baseline scans. Moreover the prostate cancer patients with the cut off $\% \mathrm{BSI}>1, \% \mathrm{PAB}>0.5, \mathrm{BLS}>5$ and EOD with grade $2,3 \& 4$ showed increased risk of disease progression and less survival.
\end{abstract}

Keywords: Bone scan; Prostate cancer; Ultra sonography; Computed tomography

\begin{abstract}
Abbreviations: MDP: Methyl-Di-Phosphonate; PET: Positron Emission Tomography; SPECT: Single Photon Emission Computed Tomography; BSI: Bone Scan Index; BLS: Bone Lesion Scoring; EOD: Extent of Disease; PAB: Positive Area on Bone Scan; PSA: Prostate Specific Antigen; CT: Computed Tomography; MRI: Magnetic Resonance Imaging; USG: Ultra Sonography; EBRT: External Beam Radiation Therapy
\end{abstract}

\section{Introduction}

\section{The skeleton}

The adult human skeleton has a total of 213 bones, excluding the sesamoid bones. The appendicular skeleton has 126 bones, axial skeleton 74 bones, and auditory ossicles six bones. Each bone constantly undergoes modelling during life to help it adapt to changing biomechanical forces, as well as remodelling to remove old, micro damaged bone and replace it with new, mechanically stronger bone to help preserve bone strength [1].

\section{Bone tumours}

Bone tumours develop when cell in the bone divide without control, forming a mass of tissue. Most bone tissues are benign and they don't spread. However they may still weaken bone and can lead to fracture and cause other problems [2]. Bone cancers may destroy normal bone tissues and can spread to other parts of the body called as metastasis (Table 1).
Benign bone tumors: They are more common than the malignant tumours. Following are the most common benign tumours.

- Osteochondroma

- Osteoid osteoma

- Giant cell tumour

- Osteoblastoma

- Enchondroma

Metastatic cancer: The metastatic bone cancer is the one in which primary is present somewhere else in the body whereas it metastasize to bone. Even though it spreads to the bone it is not considered as the bone tumour because the primary is present elsewhere. Cancers that commonly spread to the bones are:

- $\quad$ Breast cancer

*Corresponding author: Nayab Mustansar, Nuclear Physician, AFIP (Armed Forces Institute of Pathology), Islamabad, Pakistan; E-mail: dr.m.nayab@gmail. com

Received October 23, 2018; Accepted November 27, 2018; Published December 04, 2018

Citation: Mustansar N (2018) Utility of Bone Scan Quantitative Parameters for the Evaluation of Prostate Cancer Patients. J Nucl Med Radiat Ther 9: 391. doi: 10.4172/2155-9619.1000391

Copyright: (c) 2018 Mustansar N. This is an open-access article distributed under the terms of the Creative Commons Attribution License, which permits unrestricted use, distribution, and reproduction in any medium, provided the original author and source are credited. 
Citation: Mustansar N (2018) Utility of Bone Scan Quantitative Parameters for the Evaluation of Prostate Cancer Patients. J Nucl Med Radiat Ther 9: 391. doi: 10.4172/2155-9619.1000391

Page 2 of 11

\begin{tabular}{|c|c|c|c|c|}
\hline Primary Tumour & $\begin{array}{c}\text { Incidence of Bone } \\
\text { Metastasis }\end{array}$ & $\begin{array}{c}\text { Incidence of Bone Metastasis in Advanced } \\
\text { Disease (At Autopsy) }\end{array}$ & $\begin{array}{c}\text { Median Time of Survival after Diagnosis of } \\
\text { Bone metastasis }\end{array}$ & $\begin{array}{c}\text { Five Year World } \\
\text { Prevalence }\end{array}$ \\
\hline Breast & 73 & $65 \%-75 \%$ & $19-25$ months \\
\hline Prostate & 68 & $65 \%-75 \%$ & $12-53$ months \\
\hline Thyroid & 42 & $60 \%$ & 48 months \\
\hline Kidney & $20-25$ & & 6000 \\
\hline Lung & 36 & $30 \%-40 \%$ & $7,555,000$ \\
\hline GIT & 5 & & 475,000 \\
\hline Myeloma & & $70 \%-95 \%$ & $7,394,000$ \\
\hline Melanoma & & $14 \%-45 \%$ & $6-54$ months \\
\hline
\end{tabular}

Table 1: Incidence of bone metastasis, prevalence and survival [2]

- Prostate cancer

- Lung cancer

\section{Importance of PSA (prostate specific antigen)}

Prostate specific antigen is the only antigen yet reported which has relatively prostate specificity [3]. Monoclonal antibodies that recognize the extracellular domain of PMSA have recently been reported and are currently being evaluated for use in prostate cancer and treatment [4]. It is a specific tumour marker for the prostate cancer and is clinically valuable marker for prostatic adenocarcinoma in the initial evaluation of the patient. Although the serum PSA levels appear to be an important and independent prognostic factor with its interaction with rest of the factors like clinical staging, Grading and nodal status. Pretreatment PSA levels are an important in the outcome of the localized prostate cancer treated with the external beam radiation therapy.

\section{Materials and Methods}

The study was conducted at N.O.R.I (Nuclear Medicine Oncology and Radiotherapy Institute, Islamabad) from October 2013 to April 2014.

\section{Patient's demographic data}

Total 141 patients with baseline bone scans were included in the study. The characteristics of the study population are depicted in the Table 2.

The patients with both baseline and follow up scans were 40 in total with median age of 77 and median PSA level of 200. The demographic data is given in the Table 3 .

- Study design: Cross sectional study.

- $\quad$ Place of study: Nuclear medicine department of NORI.

- Duration: Six months.

- $\quad$ Sampling Method: Non probability purposive sampling method.

- Sample size: 141 Patients with histopathologically proved prostate cancer. (Baseline and Follow up scan of 40 patients with hormonal treatment)

\section{Sample selection}

\section{Inclusion criteria:}

1. Histological confirmed prostate cancer patients referred for evaluation of osseous metastasis within three months of diagnosis.

2. Written informed consent.

\begin{tabular}{|c|c|c|c|c|c|}
\hline \multicolumn{2}{|c|}{ Variable at Baseline } & No. of Patients & $\%$ & Median & Range \\
\hline Age, Years & & 141 & & 75 & 24 \\
\hline PSA ng/ml & & 141 & & 380 & 1236 \\
\hline \multirow{2}{*}{ Patient Status } & Dead & 21 & 14.89 & & \\
\cline { 2 - 6 } & Alive & 120 & 85.11 & & \\
\hline
\end{tabular}

Table 2: Patient's demographic data (baseline bone scan).

\begin{tabular}{|c|c|c|c|c|c|}
\hline \multicolumn{2}{|c|}{ Variable at Baseline } & No. of Patients & $\%$ & Median & Range \\
\hline Age, Years & & 40 & & 77 & 23 \\
\hline PSA ng/ml & & 40 & & 200 & 1614 \\
\hline \multirow{2}{*}{ Patient Status } & Dead & 13 & 32.50 & & \\
\cline { 2 - 6 } & Alive & 27 & 67.50 & & \\
\hline
\end{tabular}

Table 3: Patient's demographic data (baseline and follow up scans).

\section{Exclusion criteria:}

1. $<18$ years.

2. Patients in which PSA levels was not available

3. Patients having other co-morbids.

Every patient included in our study underwent Bone Scintigraphy and later the quantitative parameters were applied on the bone scan. We have applied different methods of quantitative parameters for the assessment of tumour burden on the bone scan baseline and follow up bones can. These methods include:

- $\quad \%$ BSI (bone scan index).

- $\quad$ Extent of Disease (EOD)

- $\quad$ \%AB (Positive areas on bone scan).

- Bone Lesion Scoring (BLS) Method.

Following quantitative parameters were applied on the bone scans:

\%BSI (Bone scan index) method: \%BSI is one of the most frequently used quantitation method and is also available as commercial software. Based on ICRP Publication No.23, 158 bones were listed by name and the weight of each bone was expressed as a fraction of the weight of the entire skeleton. The fractional involvement of each bone was calculated subjectively on each bone scan. BSI was calculated by summing the product of the weight and the fractional involvement. So it has been carried out manually by designing a BSI calculator in Microsoft excel such that the weight in grams of each bone is multiplied with the percentage of the bone involvement and then is divided by the total percentage of the respective bone i.e. 100\% (Figure 1).

Formula used:

$$
\text { Bone Involvement }=\frac{\text { Total weight in grams } \times \text { involvement of bone }}{\text { Total percentage of bone }}
$$

$=\mathrm{ABC}(\mathrm{Grams})$ 
Citation: Mustansar N (2018) Utility of Bone Scan Quantitative Parameters for the Evaluation of Prostate Cancer Patients. J Nucl Med Radiat Ther 9: 391. doi: 10.4172/2155-9619.1000391

Page 3 of 11

$$
\begin{aligned}
\% B S I= & \frac{\text { boneinvolvement }(\text { grams }) \times 100}{\text { Total skeletal weight in grams }} \\
& \text { Total weight male }=5500 \mathrm{gms}
\end{aligned}
$$

Percentage of total fresh skeletal mass in grams used for calculation is shown in the Table 4 [ICRP-23] [5].

This method of quantitation was applied to 141 patient's baseline scan and also to 40 patients with both the baseline and follow up scans. Value of 1 was used as cut off, below that patients were considered low risk for disease progression and above that were considered as high risk for disease progression. This value was chosen based on the previously published study in which the cut off was used for the purpose of calculation.

Extent of disease (EOD)-grading: This method of quantitation is based on the subjective assessment of osseous metastasis on the bone scan the number of osseous metastasis is labeled as a specific grade, grade 0 means no evidence of bone metastasis, and grade 1 to 4 represents increasing osseous metastasis respectively as mentioned in Table 5

The method of calculating 'Extent of Disease' is subjective assessment of bone metastasis on skeletal scintigraphy. The number of lesions determines the extent of disease as explained in the table above. The arbitrary cut off for the purpose of quantitation and analysis of survival was taken as grade $0 \& 1$ and grade $2,3 \& 4$. The patients with grade $0 \& 1$ were considered low risk whereas those with grade 2,3 \& 4 were considered high risk. Soloway et al. used the same method for the bone metastasis quantitation and survival analysis. Extent of DiseaseGrading is shown in Figure 2.

\%PAB (Positive area on bone scan): Positive area on bone scan is a quantitative method in which the osseous metastasis is considered as the positive area. The same method was applied to the dataset of patients using the formula given below:

$$
\% P A B=\frac{\text { Positive on bone scan } \times 100}{\text { Square area }[\text { Width of gluteal region } \times \text { Height of entire skeleton }]}
$$

In this method the involved areas on the bone scan of a patient were measured using the computer software and are summed up; used as a numerator as mentioned in the above formula, whereas using the same software the width of the hip bone and the height of the skeleton was measured on the same bone scan; using as a denominator as per

\begin{tabular}{|c|c|c|}
\hline Regions & Weight in Grams & \%age weight \\
\hline Head: Skull & 649 & 11.80 \\
\hline Mandible & 66 & 1.20 \\
\hline $\begin{array}{c}\text { Trunk: Vertebrae } \\
\text { Sacrum }\end{array}$ & 66 & 1.20 \\
\hline Ribs & 385 & 7.00 \\
\hline Sternum & 66 & 1.20 \\
\hline Limbs: Femora & 842 & 15.30 \\
\hline Tibiba \& Fibula & 622 & 11.30 \\
\hline Pelvic Bones & 583 & 10.60 \\
\hline Feet & 346 & 6.29 \\
\hline Humeri & 292 & 5.30 \\
\hline Radii \& Ulana & 198 & 3.60 \\
\hline Scapula & 198 & 3.60 \\
\hline Hands & 127 & 2.30 \\
\hline Clavicle & 44 & 0.80 \\
\hline Patella & 39 & 0.70 \\
\hline
\end{tabular}

Table 4: Percentage of total fresh skeletal mass in grams.

\begin{tabular}{|c|c|}
\hline Grade & Extent of Disease \\
\hline Grade -0 & No Metastasis \\
\hline Grade- 1 & $<6$ Bone Mets, Vertebral Body $=2$ \\
\hline Grade-2 & $6-20$ Bone Mets \\
\hline Grade-3 & $>20$ Bone Mets but $<$ Super Scan \\
\hline Grade-4 & Super Scan \\
\hline
\end{tabular}

Table 5: Extent of disease (Grading).

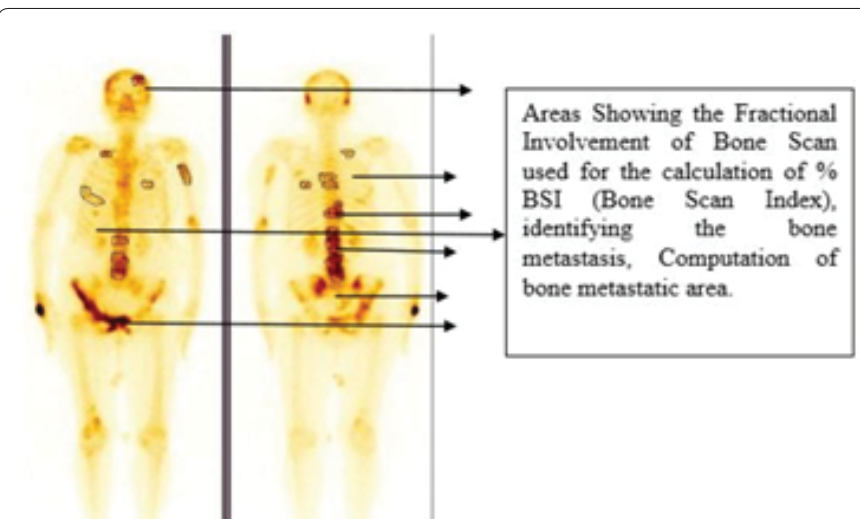

Figure 1: Fractional involvement of bone due to metastasis and BSI calculation.

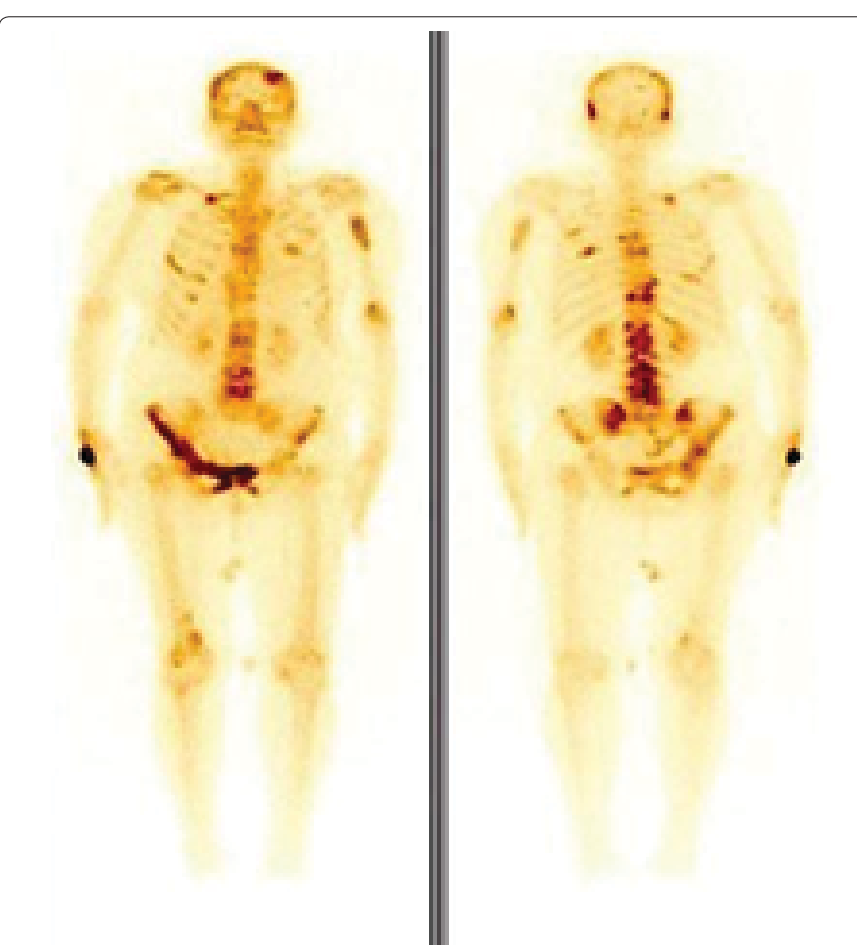

Figure 2: Scan showing multiple metastasis in the region of skull, spine, pelvis and long bones.

mentioned in formula and finally the percentage was calculated. The arbitrary cut off for \% $\mathrm{PAB}$ method was taken as 0.5 .The patients with $\% \mathrm{PAB}$ above this cut off values were considered high risk as compared to the dataset of patients having \% $\mathrm{PAB}$ values below this cut off. Nogouchi et al. used the same method of \%PAB for osseous metastasis quantitation and survival analysis. \% PAB Calculation was calculated 
Citation: Mustansar N (2018) Utility of Bone Scan Quantitative Parameters for the Evaluation of Prostate Cancer Patients. J Nucl Med Radiat Ther 9: 391. doi: 10.4172/2155-9619.1000391

Page 4 of 11

using following steps using computer software (Figures 3 and 4 ).

Bone lesion scoring: The bone lesion scoring is also a subjective method in which numbers of lesions are assessed clinically at different regions of the skeleton and is then summed up to find out the exact scoring of the scan (Proposed by Prof. Guiliano Mariani, Universtiy di Pisa Italy, verbal Communication).The following regions have been given score as shown in the Table 6.

The cut off for BLS was taken arbitrarily as 5 for the purpose of survival analysis and prognosis evaluation. The patients with baseline bone scan bone lesion scoring (BLS) $>5$ were considered as high risk whereas those with BLS $<5$ were considered low risk.

\section{Results}

\section{Comparing \%BSI quantitation method with PSA levels}

By applying correlation statistics (goodness of fit model) we compared the relationship of \%BSI and PSA levels (Figure 5).

Where $\mathrm{Y}$ is the dependent Variable i.e. BSI; PSA is the independent or explanatory variable. $\mathrm{X}$ is the slope/beta which shows the rate of the change in the variable. Alpha i.e. constant 0.9423 is the intercept. $\mu \mathrm{t}$ is the error term.

$R^{\wedge} 2$ goodness and fitness of the model: If $R \wedge 2$ value is near to 1 or equal to 1 it shows that model is best fitted. Here $\mathrm{R}^{\wedge} 2$ value is 0.891 which shows the goodness of the model. This shows that as the PSA level increases the \%BSI quantitation values also increases, showing the linear relationship between the dependent and independent variables. Thus proving the linear relationship between the BSI and PSA levels. The $\%$ BSI values of the data set can be represented via bar diagram in Figure 6 .

\section{Comparing \% PAB quantitation method with PSA levels}

Similarly correlation statistics was also applied on the \% $\mathrm{PAB}$ quantitation method and PSA levels to see the relationship between the two variables (Figure 7).

Equation derived from the graph:

$$
\begin{aligned}
& Y=\alpha+\beta X t+\mu t \\
& Y=0.001 x+0.2677
\end{aligned}
$$

Here $\mathrm{R}^{\wedge} 2$ value is 0.9287 which shows excellent linear correlation between \% $\mathrm{PAB}$ and PSA levels, concluding the linear increase in quantitative values of the $\% \mathrm{PAB}$ method with PSA levels. Bar diagram

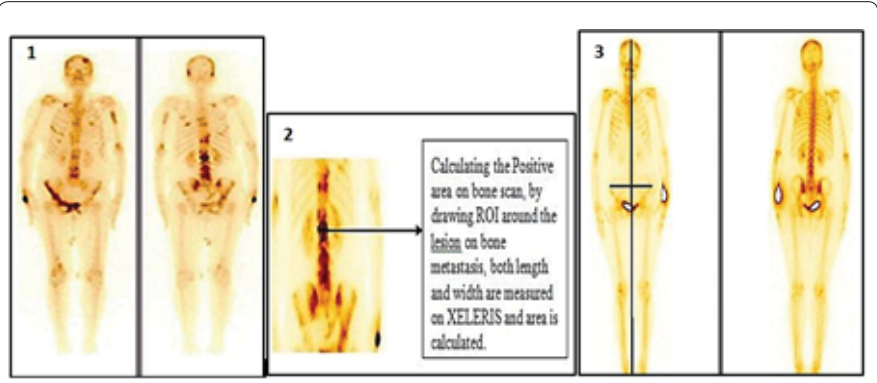

Figure 3: \% PAB calculations; Step 1: Calculating the positive area on bone scan, Step 2: Calculating the height of the skeleton along with the width from the gluteal region on the Scan using same XELERIS software in $\mathrm{mm}$ units. (The Height is taken from vertex till heel of the skeleton whereas the width is calculated using the bilateral anterior superior ileac spine), Step 3: Calculating the $\%$ PAB of the scan by applying the formula.

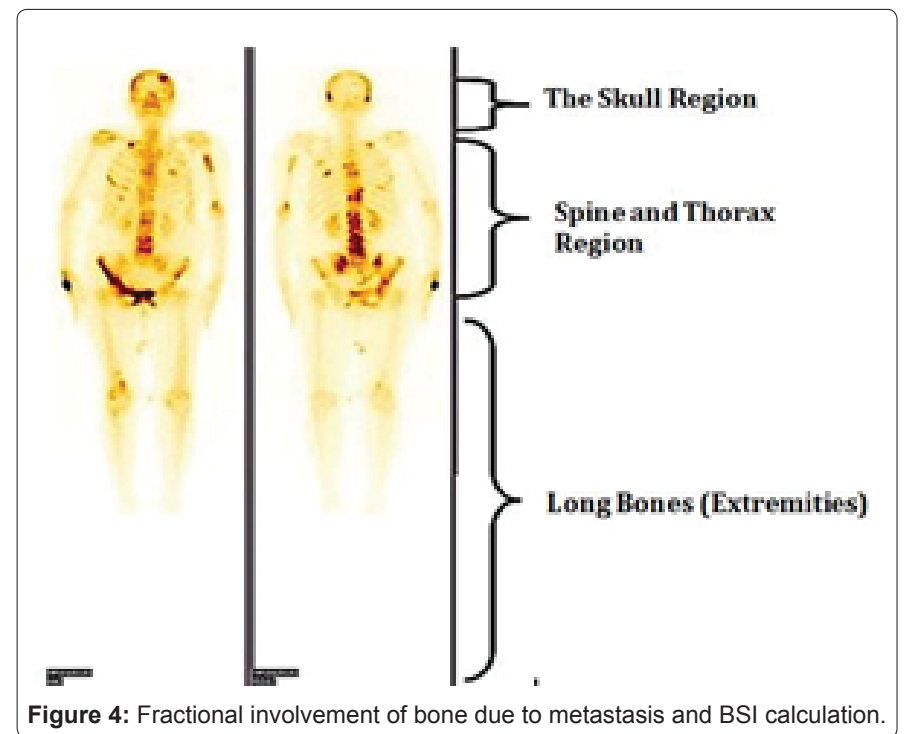

\begin{tabular}{|c|c|c|c|c|c|}
\hline Scoring & $\begin{array}{c}\text { Skull } \\
\text { Metastasis }\end{array}$ & $\begin{array}{c}\text { Spine } \\
\text { Metastasis }\end{array}$ & Pelvis & Thorax & Extremities \\
\hline 0 & No Mets & No Mets & No Mets & No Mets & No Mets \\
\hline 1 & $<$ or $=2$ & $<$ or $=2$ & $<$ or $=10 \%$ & $<$ or $=2$ & $<$ or $=2$ \\
\hline 2 & $>2$ & 3 to 5 & $10-25 \%$ & 3 to 5 & 3 to 5 \\
\hline 3 & - & $>5$ & $>25 \%$ & $>5$ & $>5$ \\
\hline \multicolumn{5}{|c}{ Table 6: Bone lesion scoring. } \\
\hline
\end{tabular}
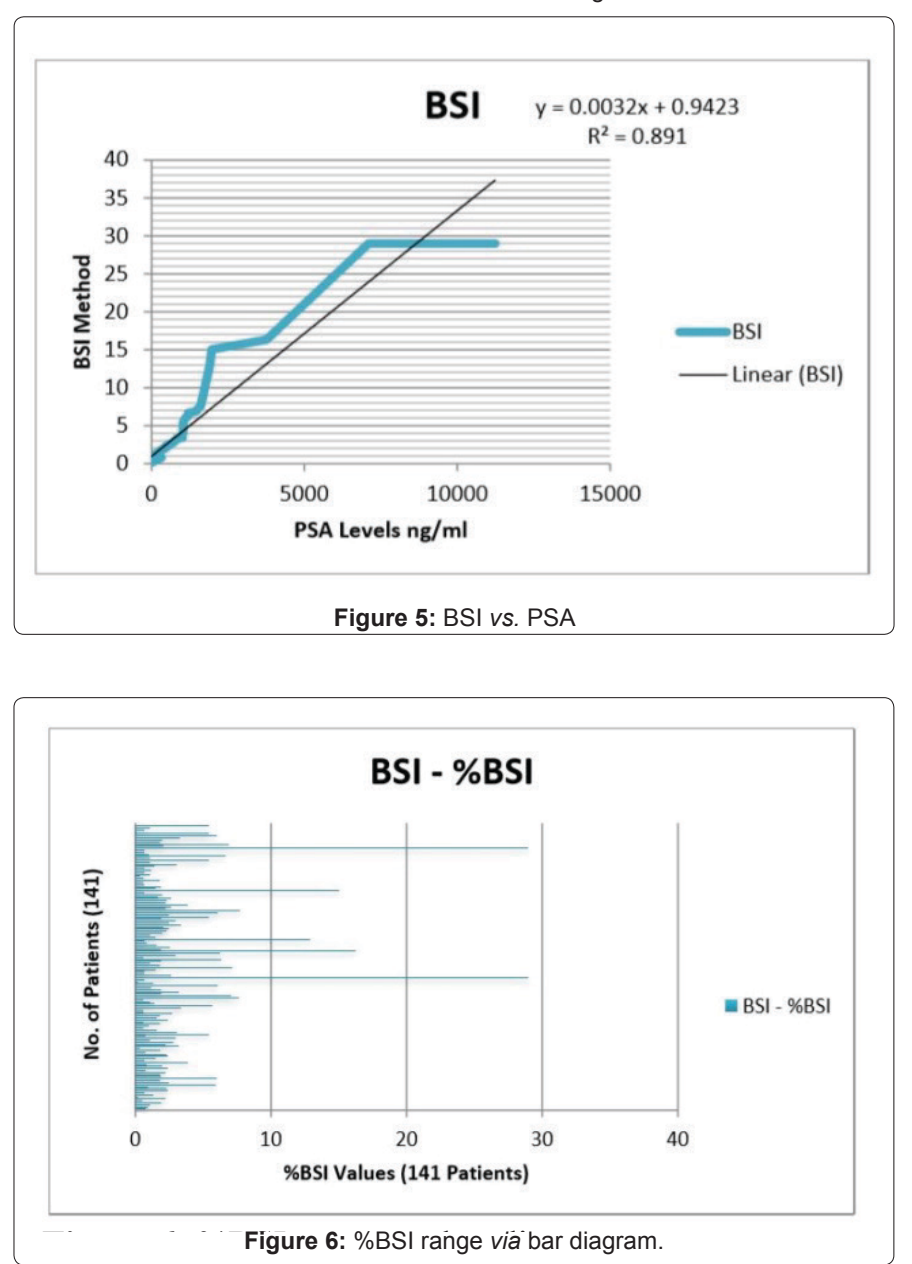

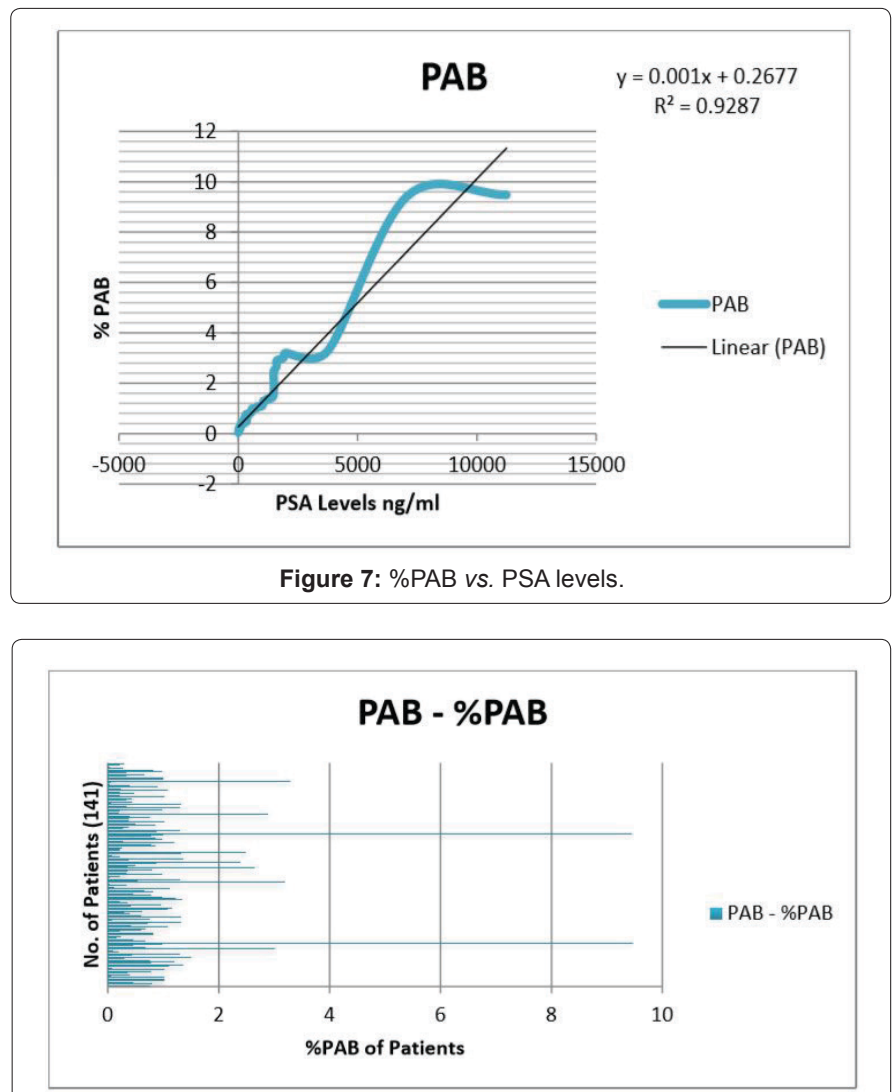

Figure 8: \% PAB range via bar diagram.

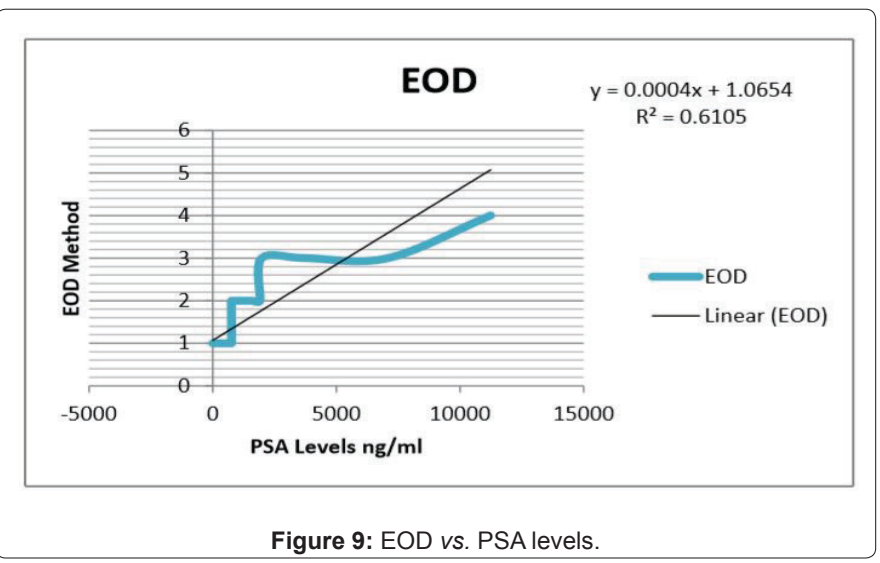

representing the \%PAB values of the data set is shown in Figure 8.

\section{Comparing EOD quantitation method with PSA levels}

The EOD method of quantitation was compared with PSA levels by using correlation statistics to see the response and general trend of the two variables (Figure 9).

Hence it can be concluded PSA level shows variability with extent of disease quantitation method $\left(\mathrm{R}^{\wedge} 2 \mathrm{0.6105}\right)$, so it was observed that as the PSA level increases the quantitative method values did not increases linearly. Data set with their respective EOD values can be represented via bar diagram as shown below, representing that the maximum bulk of patients were having grade 0,1 and 2 extent of disease with few showing grade 3 and 4 in Figure 10.
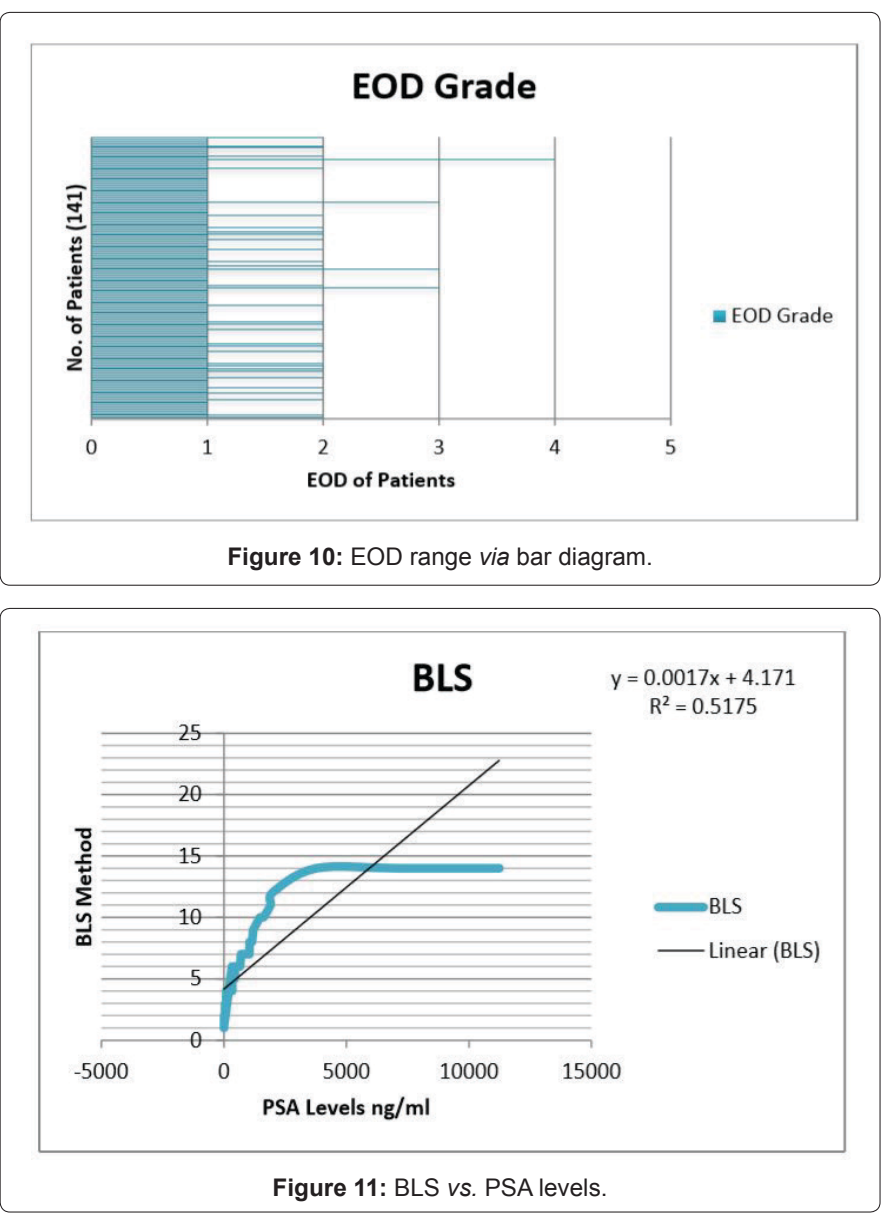

\section{Comparing BLS quantitation method with PSA levels}

Correlation statistics was applied to see the relationship between BLS quantitation method and PSA levels as mentioned in the Figure 11.

$\mathrm{R}^{\wedge} 2$ value was calculated as 0.5175 . Hence it can be concluded from the $\mathrm{R}^{\wedge} 2$ value that the increasing BLS quantitation method shows variability with the rising PSA levels. This means there is not good linear correlation between BLS quantitation method and PSA levels. We have applied this method of quantitation on 141 scans; the data can be represented using Bar chart, showing the BLS Method value in every patient (Figure 12).

As we applied quantitation methods on the baseline scans of 141 patients and later correlated them with the PSA Levels. So PSA levels of the whole population under consideration can be represented as follows using bar chart in Figure 13.

Analysis of baseline and follow up scan in dead vs. alive patients using four quantitative parameters

The baseline and follow up scans were analyzed by using quantitative parameters as follows:

Extent of disease in baseline and follow up scan and effect on survival: The EOD was applied to the baseline and follow up scans of the 40 patients. It was observed that the patients with the increase in tumour burden in follow up scans died whereas the patients with tumour burden in follow up scans showed better survival. It can be represented as shown in the Table 7. 


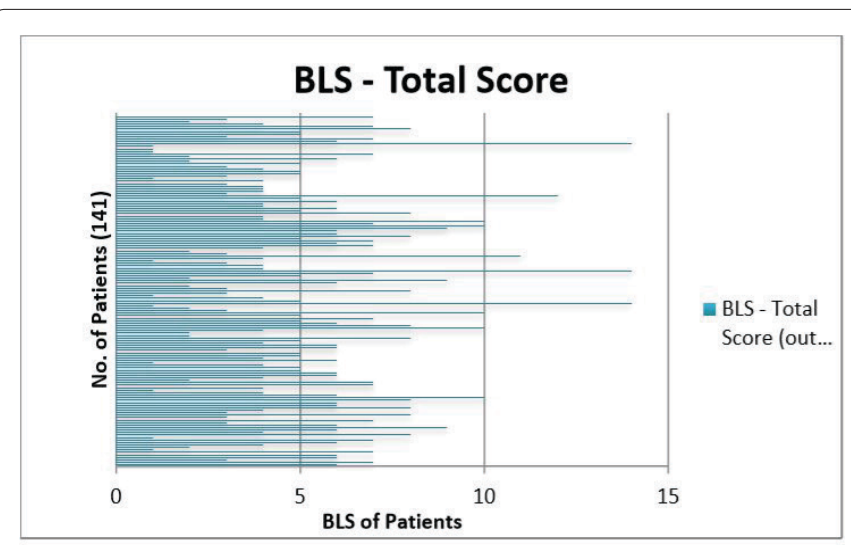

Figure 12: BLS range via bar diagram.

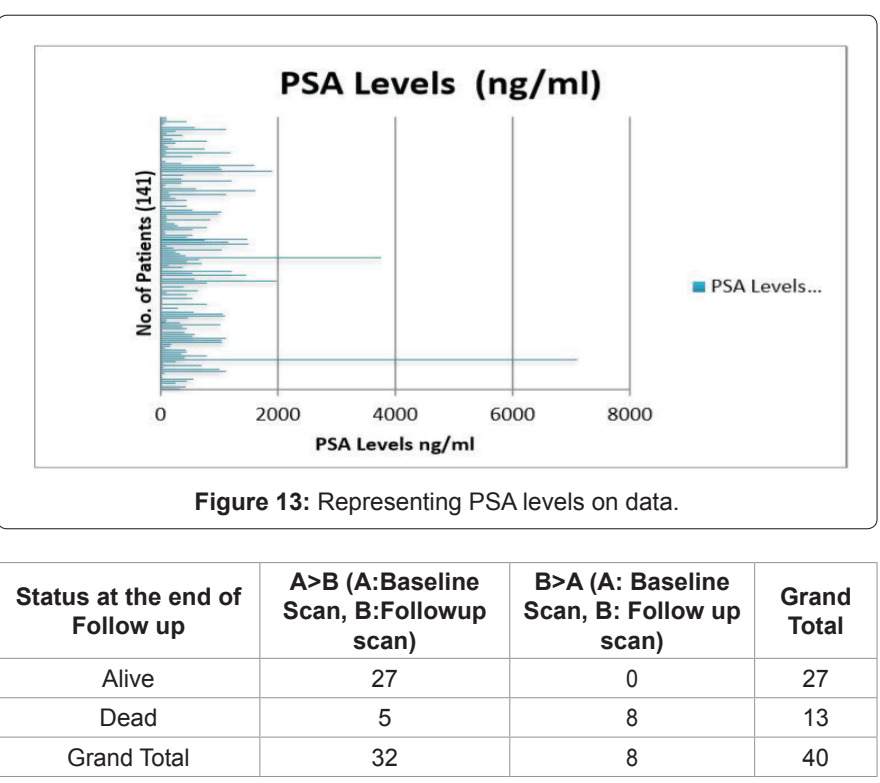

Table 7: Effect of EOD calculations in baseline and follow up scans.

The 'A' represents the tumour burden (EOD) on baseline bone scan whereas the 'B'represents the tumour burden (EOD) on follow up bone scans. By comparing the baseline and follow up scan with the extent of disease it has been observed that as the tumour burden increases in follow up scans there is less chances of the survival of a patient. By this method we conclude from above table that out of 13 dead patients this method significantly tells the rate of decrease survival i.e. $61.53 \%$

By using the cut off of grade $0 \& 1$ and grade 2,3 \& 4.It was further observed that the patients with the increase in tumour burden specifically with EOD-Grade 2, $3 \& 4$ in follow up scans died as compared to the patients with tumour burden with EOD-Grade 0 \& 1 in follow up scans. It can be represented via bar diagram as in Figure 14.

As shown in Figure 14 the patients with the increased tumour burden EOD-grade 2, $3 \& 4$ after treatment in the follow up scans (red bars) died whereas the patients with less tumour burden EOD-0 \& 1 after treatment in the follow up scans (Red bars) showed good survival.

Bone lesion scoring in baseline and follow up scan and its effect on survival: The quantitative parameter of BLS was applied to the data set of 40 patients. The patients with the increase tumour burden on follow up scans after treatment showed bad response to the treatment

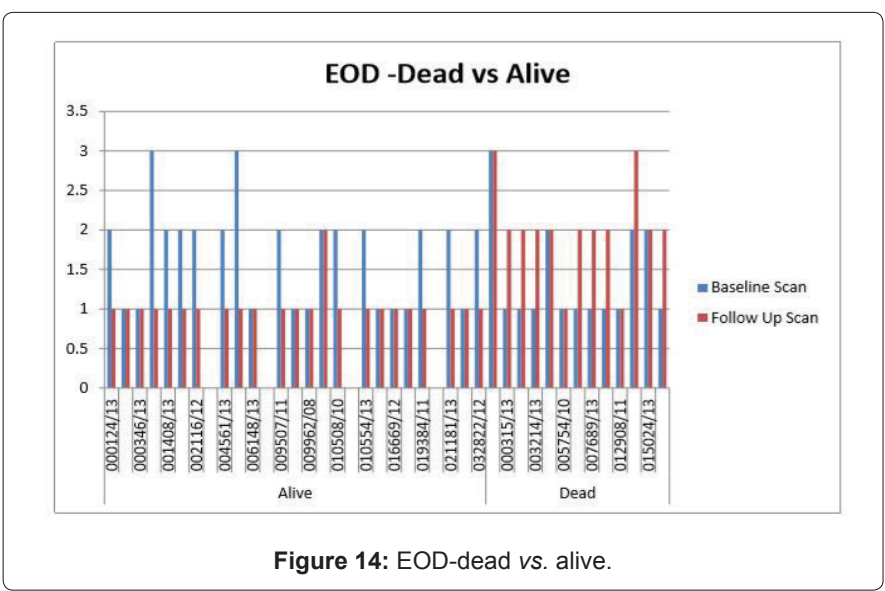

\begin{tabular}{|c|c|c|c|}
\hline $\begin{array}{c}\text { Status at the end of } \\
\text { Follow up }\end{array}$ & $\begin{array}{c}\text { A>B (A:Baseline } \\
\text { Scan, B:Followup } \\
\text { scan) }\end{array}$ & $\begin{array}{c}\text { B>A (A: Baseline } \\
\text { Scan, B: Follow } \\
\text { up scan) }\end{array}$ & Grand Total \\
\hline Alive & 26 & 1 & 27 \\
\hline Dead & 1 & 12 & 13 \\
\hline Grand Total & 27 & 13 & 40 \\
\hline
\end{tabular}

Table 8: Effect of bone lesion scoring in baseline and follow up scans.

and ultimately death. Whereas it has been observed that the patients with the decrease tumour burden on follow up scans showed good survival and better treatment response (Table 8 ).

The 'A' represents the tumour burden BLS on baseline bone scan whereas the 'B'represents the tumour burden on follow up bone scan. By comparing the tumour burden via BLS quantitative parameter on baseline and follow up scans it has been observed that as the tumour burden increases in follow up scans there was less chances of the survival of a patient and thus showing treatment response failure i.e. $92.3 \%$. Furthermore by using the cut off of 5 in BLS method it has been observed that the patients with the BLS values $>5$ on follow up scans after treatment showed bad response to the treatment and ultimately death. Whereas the patients with the BLS values $<5$ showed good survival and better treatment response. It can be represented via bar diagram as in Figure 15.

From the bar diagram it was concluded that in data of alive patients (shown on the left extreme side) the tumour burden as calculated via BLS method, was more in the baseline scan (represented as blue bars) which later after treatment, decreases $($ BLS $<5)$ in the follow up scan (represented as red bars) suggesting increase in survival of these patients. Whereas the extreme right of the bar diagram shows dead patients data suggesting that the baseline scan calculated tumour burden (represented as blue bars) did not improve after treatment showing increase in tumour burden BLS $>5$ (represented as red bars) thus showing the treatment failure and ultimately death.

\%BSI in baseline and follow up scan and its effect on survival: The \%BSI quantitative method was applied on the baseline and follow up scans of the patients. It is represented in the Table 9.

The 'A' represents the tumour burden on baseline bone scan whereas the 'B'represents the tumour burden on follow up bone scan. By comparing the baseline and follow up scan with the bone scan index (\%BSI method) it has been observed that as the tumour burden increases in follow up scans after treatment there was less chances of the survival of a patient. By this method we conclude from above table that out of 13 dead patients this method accurately tells that there is almost 


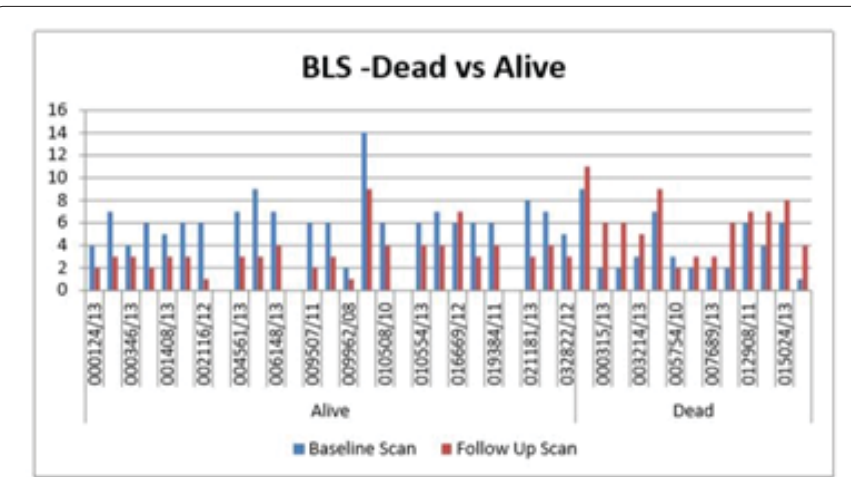

Figure 15: BLS-dead vs. alive.

\begin{tabular}{|c|c|c|c|}
\hline $\begin{array}{c}\text { Status at the end of } \\
\text { Follow up }\end{array}$ & $\begin{array}{c}\text { A>B (A:Baseline } \\
\text { Scan, B:Followup } \\
\text { scan) }\end{array}$ & $\begin{array}{c}\text { B>A (A: Baseline } \\
\text { Scan, B: Follow } \\
\text { up scan) }\end{array}$ & Grand Total \\
\hline Alive & 24 & 3 & 27 \\
\hline Dead & 0 & 13 & 13 \\
\hline Grand Total & 24 & 16 & 40 \\
\hline
\end{tabular}

Table 9: Effect of \% BSI calculation in baseline and follow up scans.

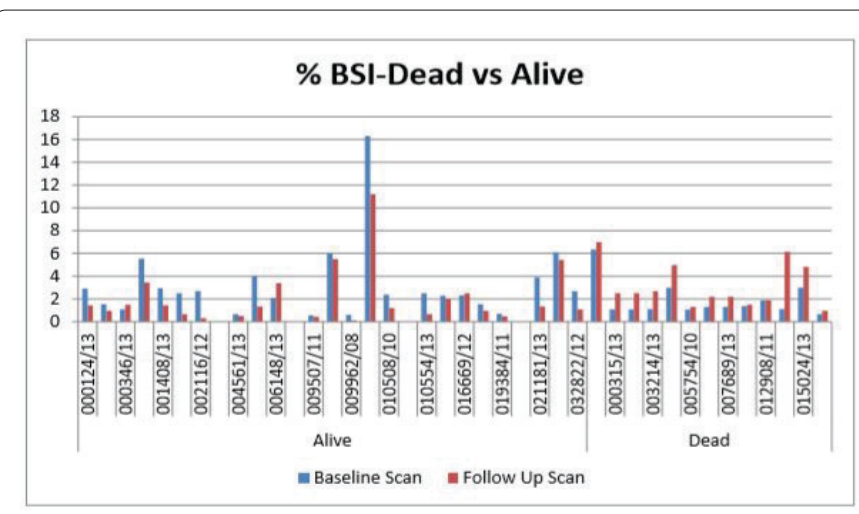

Figure 16: \%BSI-dead vs. alive.

no chance of patient survival $100 \%$ dead.

It was furthermore observed that by taking the cut off of 1 , the patient's follow up scan showing the tumour burden of $\%$ BSI $>1$ showed poor survival as compared to the patient's follow up scans with cut off values $<1$ as shown in Figure 16.

It has been observed from the bar diagram that in data of alive patients (shown on the left extreme side) the tumour burden as calculated via \%BSI method, was more in the baseline scan (represented as blue bars) which later after treatment, decreases in the follow up scan (represented as red bars) suggesting good response to the treatment and thus increase survival of these patients. Whereas the extreme right of the bar diagram shows dead patients data suggesting that the baseline scan calculated tumour burden (represented as blue bars) did not improve after treatment showing increase in tumour burden with \%BSI $>1$ (represented as red bars) thus showing the treatment failure and less survival.

\% PAB in baseline and follow up scan and its effect on survival: Similarly the \% PAB quantitative parameter was applied on the baseline and follow up scans of 40 patients. It was observed that increase in the tumour burden \% PAB on the follow up scans after treatment is a bad prognostic factor (Table 10).

\begin{tabular}{|c|c|c|c|}
\hline $\begin{array}{c}\text { Status at the end of } \\
\text { Follow up }\end{array}$ & $\begin{array}{c}\text { A>B (A:Baseline } \\
\text { Scan, B:Followup } \\
\text { scan) }\end{array}$ & $\begin{array}{c}\text { B>A (A: Baseline } \\
\text { Scan, B: Follow } \\
\text { up scan) }\end{array}$ & Grand Total \\
\hline Alive & 25 & 2 & 27 \\
\hline Dead & 1 & 12 & 13 \\
\hline Grand Total & 26 & 14 & 40 \\
\hline
\end{tabular}

Table 10: Effect of \% PAB calculation in baseline and follow up scans

The 'A' represents the tumour burden on baseline bone scan whereas the 'B'represents the tumour burden on the follow up bone scan. By comparing the tumour burden on baseline and follow up scan with the \%PAB ( Positive area on bone scan) it has been observed that as the tumour burden increases in follow up scans there is less chances of the survival of a patient. By this method we conclude from above table that out of 13 dead patients this method significantly tells the rate of decrease survival i.e. $92.3 \%$.

Furthermore by taking the cut off of 0.5 , it was observed that the patients with tumour burden $>0.5$ in follow up bone scans showed treatment failure and disease progression as compared to the patients with the less tumour burden $\% \mathrm{PAB}$ value of $<0.5$. It can be represented via bar diagram as in Figure 17.

It has been observed from the bar diagram that in data of alive patients (shown on the left extreme side) the tumour burden as calculated via \% $\mathrm{PAB}$ method, was more in the baseline scan (represented as blue bars) which later after treatment, decreases in the follow up scan (represented as red bars) suggesting increase in survival of these patients. Whereas the extreme right of the bar diagram shows dead patients data suggesting that the baseline scan calculated tumour burden (represented as blue bars) did not improve after treatment showing increase in tumour burden $>\% \mathrm{PAB}>0.5$ (represented as red bars) thus showing the treatment failure and less survival.

PSA levels correlation with tumor burden in baseline and follow-up scan and its effect on survival: The 'A' represents PSA values of the baseline bone scan whereas the 'B'represents the PSA values of the follow up bone scan. By comparing the PSA levels of baseline and follow up scans it has been observed that as the tumour burden increases in follow up scans the PSA levels also increases showing less chances of the survival of a patient. So we conclude from above table that out of 13 dead patients, the increase in PSA levels in the follow up scans accurately tells that there is almost no chance of patient survival $100 \%$ dead (Table 11).

Correlation of PSA levels in both baseline and follow up scans of alive and dead patients with all four quantitative parameters: From the above line diagram the general trend of all the quantitative parameters with respect to PSA levels are very obvious in dead patients. It is observed that the baseline bone scans have low values as compared to the follow up scans (extreme right sided) which are calculated after treatment. Moreover the PSA levels taken during the follow up bone scan also shows marked increase relative to the PSA levels taken before baseline bone scans, signifying that increase in PSA levels in follow up scan and a similar trend of increase values of all the four quantitative parameters results in increase risk and decrease survival of the patient. Similarly following observation was made in the baseline and follow up scans of patients who were alive (Figure 18).

From the above line diagram the general trend of all the quantitative parameters with respect to PSA levels are very obvious in alive patients, which is opposite to that of the patients who were dead. It was observed that the baseline bone scans have high values as compared to the follow 


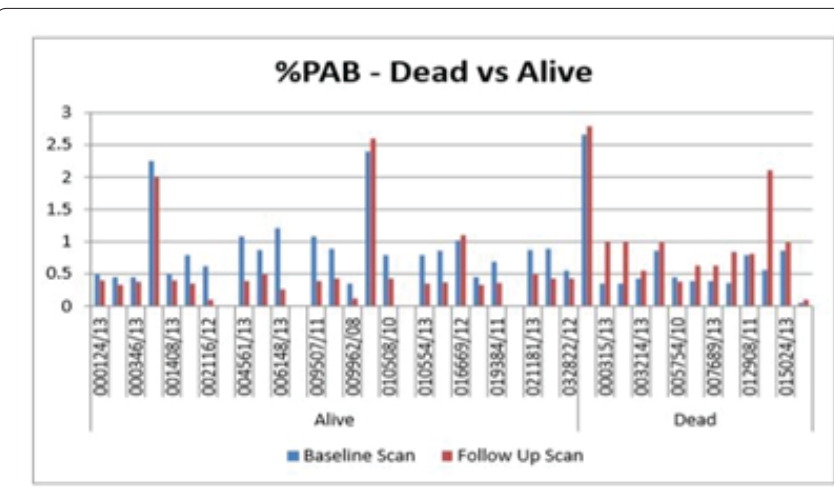

Figure 17: \% PAB-dead vs. alive.

\begin{tabular}{|c|c|c|c|}
\hline $\begin{array}{c}\text { Status at the end of } \\
\text { Follow up }\end{array}$ & $\begin{array}{c}\text { A>B (A:Baseline } \\
\text { Scan, B:Followup } \\
\text { scan) }\end{array}$ & $\begin{array}{c}\text { B>A (A: Baseline } \\
\text { Scan, B: Follow } \\
\text { up scan) }\end{array}$ & Grand Total \\
\hline Alive & 26 & 1 & 27 \\
\hline Dead & 0 & 13 & 13 \\
\hline Grand Total & 26 & 14 & 40 \\
\hline
\end{tabular}

Table 11: PSA correlation with tumour burden on serial scans.

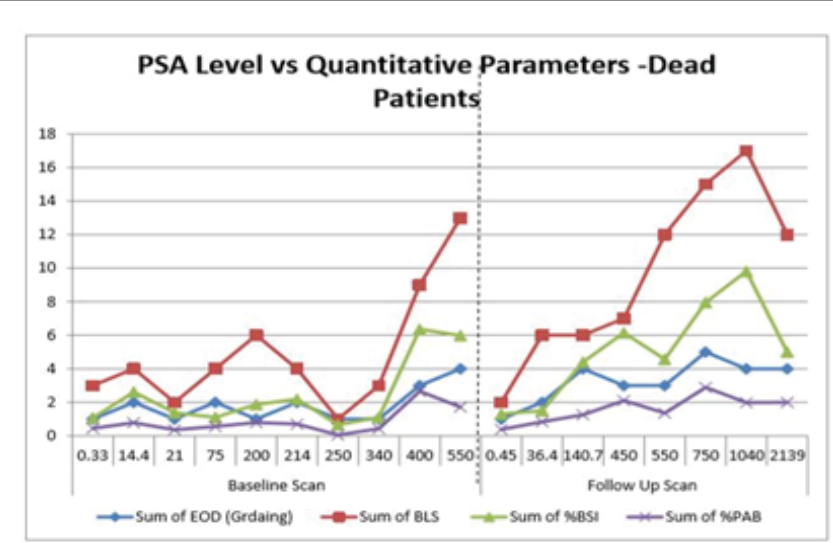

Figure 18: PSA level vs. quantitative parameters (in dead patients).

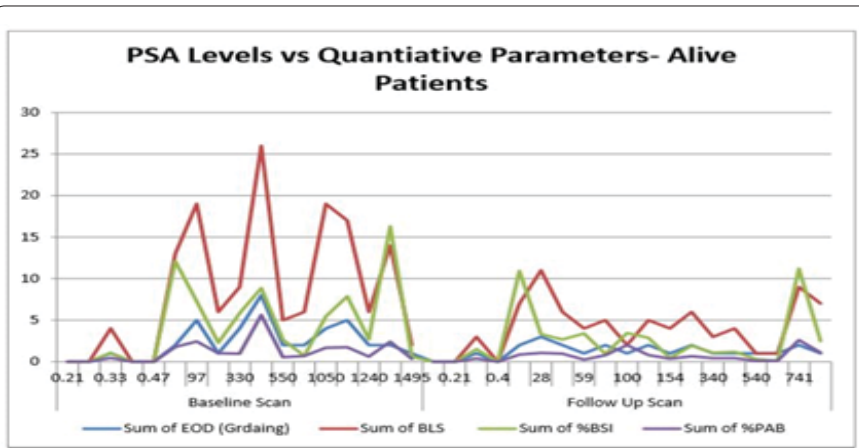

Figure 19: PSA level vs. quantitative parameters (in alive patients)

up scans (extreme right sided). Moreover the PSA levels taken during the follow up bone scan also shows marked decrease relative to the PSA levels taken before baseline bone scans, signifying that decrease in PSA levels in follow up scan and a similar trend of decrease values of all the four quantitative parameters results in good and disease free survival of the patient (Figure 19).

\section{Discussion}

The bone metastasis is one of the commonest complications of few tumours. The tumours that most commonly metastasize to bone are the tumours from the prostate in men and breast in women. Among the complication of bone metastasis, bone pain, is the worst consequence, affecting $66 \%$ of the patients who have the bone metastasis [6].

The patients with prostate cancer usually don't have any clinically measurable or evaluable method for the quantification of their tumour burden by conventional criterion. Metastasis to the bone is the most serious complication of solid malignant neoplasm, and by far the most common malignant tumour involving the skeleton [7].

The present knowledge of quantifying the metastatic bone disease is still not sufficient. A lot of work has been done to quantify the bone metastasis using bone scans. Radionuclide bone scans are strongly positive in cases of bone involvement of prostate cancer patients irrespective of whether the lesions are lytic, mixed or pure blastic. The conventional method of calculating the bone metastasis is by combining qualitative assessment of all the sequential bone scans and bony films with tumour markers (Such as PSA and Alkaline Phosphatase levels). Although serum acid phosphatase may present the progression and regression of disease, approximately $25 \%-0 \%$ of patients with metastatic disease have a normal acid phosphatase. Thus it cannot be used to tell about the tumour status [8]. Interpretation of bone scan is a subjective process, dependent on the experience and knowledge of the nuclear medicine physician is a question.

To quantitate all bone metastasis in patients is a time consuming task, since the patients with metastatic involvement usually have more than one disease site. Several studies have evaluated different ways to quantify the extent of bone involvement during therapy. Interpretation of the bone scans is a tedious procedure for the physicians and often leads to misinterpretation either as overestimation or underestimation of the metastasis. To minimize the risk of misinterpretation, one of the careful methods is quantitative analysis of the bone scans in order to ascertain, whether a metastatic lesion is present or not. There are several methods to-date which can be used to analyze the extent of such lesions. For example, quantitation of the bone scan i.e. \% BSI (Bone scan index) [9], \% PAB (Positive area on bone scans) [10], EOD (extent of disease) [11] and BLS (Bone lesion scoring) [12].

Among these, \%BSI has most frequently been used and validated in various studies. Novel automated software based on \%BSI quantitative calculations has been developed and is in clinical use in many nuclear medicine departments. However, automated \%BSI calculator (EXINI bone TM \& BONENAVITM) has not been extensively employed in routine nuclear medicine practice because of its high cost. Another potential risk of inaccuracy in using these automated quantitation softwares is their limited training databases as these softwares use either Swedish or Japanese patients data $[13,14]$. Despite all these shortcomings there is body of evidence that these quantitative bone scan parameters not only increase interpretation accuracy but can also serve as image biomarkers. Most of the published literature focuses on determining accuracy of either one or two parameters at a time. But to date there is not study comparing all these parameters. Based on this premise this study was designed to evaluate the accuracy of different bone scan qualitative methods namely \%BSI, \%PAB, EOD and BLS. Study also explored each quantitative parameter as a prognostic indicator in prostate cancer patients.

In our study we applied four quantitative parameters on baseline 
bone scans to assess the tumour burden of 141 histopathologically proved carcinoma prostate patients. Results of all fours quantitation methods were individually compared with PSA levels $(\mathrm{ng} / \mathrm{ml})$, to evaluate the efficacy of all these parameters as effective disease status indicator. For evaluation as a prognostic indicator, 40 patients having a serial follow up scans were chosen.

The follow up datasets were again analyzed using the same four bone scan quantitative parameters (Appendix). For purpose of quantification arbitrary cut off were applied for each parameter. Cut offs were $\%$ BSI: $<1$ low risk and $>1$ high risk, $\%$ PAB: $<0.5$ was low risk while $>0.5$ means high risk patients, EOD: grade $0 \& 1$ were considered low risk, grade 2, $3 \& 4$ were considered as high risk, and in BLS: score of $<5$ was considered low risk and $>5$ was vice versa. Age range of our study population was between 60 years -85 years in our study population with the mean age of 75 years. Most of the published data showed similar age range of age as seen in our patient cohort.

\section{Bone quantitative parameters as indicators of disease burden}

PSA level is mostly wide used tumour marker for disease status. Progression or regression of disease post treatment is evaluated on serial PSA measurements. Post treatment elevation of PSA invariably predicts tumor progression and can precede clinical evidence of the event by about 6 months. Parameters like pre and post treatment PSA, PSA doubling time, PSA nadir values, percentage of PSA decline are exhaustively investigated as disease status indicators, disease progression predictor or survival marker $[15,16]$.

Taking PSA as true indictor of disease, we investigated the relation between the PSA levels and the tumour burden in the baseline bone scan and later investigated the role of these quantitative parameters in both baseline and follow up scans. When we compared the correlation of PSA with the bone scan quantitative parameters, our study results showed that all four quantitative parameters assess the tumour burden to varying degree of accuracy but all these parameters have almost linear correlation with PSA levels. Best linear correlation was seen in $\% \mathrm{PAB}$ method where $\mathrm{R}^{\wedge} 2$ was 0.92 closely followed by \% BSI method where $\mathrm{R}^{\wedge} 2$ was $0.89 . \% \mathrm{PAB}$ method was initially evaluated by Noguchi et al. [17] and in their study they applied univariate and multivariate regression analysis and their results showed that only \% $\mathrm{PAB}$ came out be the most significant disease predictor for survival among all the variables they studied.

In the study Noguchi et al. concluded that \% $\mathrm{PAB}$ is a simple and reproducible method for estimating skeletal involvement in prostate cancer patients. Although in our study we applied ordinary least square regression analysis using PSA as dependent variable and it showed that our \% $\mathrm{PAB}$ data has $\mathrm{t}$-statistics value is within the prescribed range of -1.96 to +1.96 . Our own conclusion was that $\% \mathrm{PAB}$ method is easier to calculate as compared to \%BSI which needs rigorous calculations. The main advantage of the \% $\mathrm{PAB}$ method is its simplicity, accuracy in the measurements of region of interest and reproducibly which carefully estimates the percentage of the skeleton involving tumours in prostate cancer. As every single lesion is considered and calculated by drawing region of interest.

When comparing our PSA findings with \%BSI results it was seen that there is significant linear correlation present between these two variables with $\mathrm{R}^{\wedge} 2$ of 0.89 . Similarly OLS regression analysis showed t-statistics finding of \%BSI within the range. There is a large body of evidence about the BSI accuracy, reproducibility \& validity as a bone disease predictor, prognostic \& survival indicator and treatment response evaluator [18-
20]. BSI is most frequently used bone quantitative parameters it was first introduced by Imbriaco et al. [21]. Initially the calculation were done manually but now based of BSI calculation formulas, automated BSI software are available commercially. One reason for evaluating BSI along with other less commonly used quantitative parameters was that this automated software although commercially available but expensive in terms of developing country setting. Moreover these software use Swedish and Japanese datasets for training and comparison which may lead to erroneous results in our setting.

Our comparison of PSA with EOD and BLS showed linear correlation but of moderate degree with $\mathrm{R}^{\wedge} 2$ values of 0.61 and 0.51 respectively. EOD was compared with $\% \mathrm{PAB}$ by Noguchi et al [22] and it was concluded that \% $\mathrm{PAB}$ is significantly better bone disease predictor as compared to other quantitative parameters. EOD was one of this quantitative parameter. EOD and BLS are relatively simpler quantitation methods without using mathematical formulas. These parameters use only number of lesion and their sites; however our results consistently showed that they are less accurate when compared with the \% $\mathrm{PAB}$ and $\%$ BSI. This was confirmed in the OLS regression analysis results where $\mathrm{t}$-statistics value of EOD parameters lie outside the prescribed range showing that these results are not in full concordance with PSA values. The reason for relatively worse performance of BLS and EOD may be that there may be difficulty in visually counting individual lesions when lesions increase in number. Error may also be due to sites of lesions as lesions in the ribs are difficult to count and quantify, and assessment of lesions in the pelvis is complicated by the three-dimensional nature of the pelvic bone.

\section{Bone quantitative parameters as prognostic indicators and treatment response evaluator}

In subset of our patient population we did serial scanning to evaluate the efficacy of the four bone quantitative parameters as prognostic and survival indicators and a guide for treatment response evaluation. However, only BSI has been utilized for this purpose. Especially after the availability of the commercial BSI software there is growing research and clinical use of this Bone scan quantitation parameter in prognostic and survival analysis. Its utility is not only focused in prostate cancer patients but also there have been research studies utilizing it in all epithelial cell tumours leading to bone metastasis. In our study population of 40 patients we did serial scanning and predicted survival based on these parameters.

In our study, 40 patients underwent serial scanning and quantitation on both the baseline and follow up bone scans was done and its correlation with the PSA levels was calculated.PSA levels were taken before baseline and after follow up bone scans. It was concluded that the quantitative parameters were good in explaining tumour burden as regression or progression of disease, with decrease survival in patients having increased tumour burden in follow up scans. The patients who had tumour burden (More numerical values) of the baseline bone scan quantitative parameter then the follow up bone scan showed decrease risk of disease progression and good survival. It was further observed that the after some specific cut off value of the respective quantitative parameter there is increasing risk of disease progression. (\%BSI: 1, \% PAB: 0.5, EOD: grade 0\&1, grade 2, 3\&4 and BLS: 5).

In EOD parameters grading was done in a way that grade 0 \& 1 were taken as low risk patients and grade 2 and above were taken as high risk patients. In Tables 4 and 5 it is shown that those patient whose EOD decreased as compared to the baseline scan showed better survival as compared to those who had increased in grade of EOD on 
subsequent scan (38.4\% vs. $61.5 \%)$. In Figures $4-11$ it is noticeable that in alive patients group all the patients were either showing static disease (same grade) or had decline in grade $(\mathrm{N}=13)$. While in dead patients group 7 patients showed increase in the grade and 5 patients showed static grades. Out of these 5 patients 3 were already in high risk group but 2 patients were in low risk group i.e. group 1. This data shows that EOD can predict outcome and in patients having initial high risk grade or positive change in grade (from lower to higher) carries poor prognosis. The Chi-squared statistic of log rank test is 79.615 with associated P-value 0.000 less than 0.05 rejects null hypothesis. The conclusion therefore is that, statistically, the two survival curves differ significantly, or that the grouping variable has a significant influence on survival time. Kaplan-Meier plot shows disease-specific survival after treatment of metastatic prostate cancer for those with grade 0 \& 1 EOD and greater than grade $2(\mathrm{P}=0.00)$.

In $\% \mathrm{PAB}$ cut off was taken at 0.5 . Below that patient were considered low risk and above that it was high risk. In Tables 4-8 it is shown that those patient whose $\% \mathrm{PAB}$ values were decreased in follow up scans as compared to the baseline scan showed better survival as compared to those who had increased in \% PAB on subsequent scan $(7.6 \%$ vs. 92.3 $\%)$. In Figures $4-14$ it is noticeable that in alive patients group most of the patients were showing decline in $\% \mathrm{PAB}(\mathrm{N}=21)$. While in dead patients group 11 patients showed increase in the $\% \mathrm{PAB}$ and 1 patients showed static $\% \mathrm{PAB}$. Out of these 11 patients 4 were already in high risk group but 7 patients were in low risk group i.e. $\% \mathrm{PAB}<0.5$. However if we make our cut off point lower than many of the dead patient will shift into the high risk group. PAB was initially used by Noguchi et al and they used cut off point at 0.46 . This data shows that PAB can predict outcome and in patients having initial high risk grade or positive change in grade (from lower to higher) carries poor prognosis. The Chi-squared statistic of log rank test is 28.257 with associated P-value 0.000 less than 0.05 rejects null hypothesis. The conclusion therefore is that, statistically, the two survival curves differ significantly, or that the grouping variable has a significant influence on survival time. The conclusion is that the curve representing the patients with the decrease tumour burden $(<0.5)$ has low risk and better survival then the patients with $\% \mathrm{PAB}$ values $>0.5$. Similar findings were noted by Noguchi as well in 56 patients which they analyzed. Kaplan-Meier plot shows diseasespecific survival after treatment of metastatic prostate cancer for those with $\% \mathrm{PAB}<0.5$ and greater than 0.5 ( $\mathrm{P} 0.00)$.

For $\%$ BSI 1 was taken as cut off. Below that patient were considered low risk and above that it was high risk. In Tables 4-7 it is shown that those patient whose \%BSI decreased as compared to the baseline scan showed better survival as compared to those who had increased in \% BSI on subsequent scan (0\% vs $100 \%)$. In Figures 4-13 it is noticeable that in alive patients group most of the patients were showing decline in \%BSI $(\mathrm{N}=19)$. While in dead patients group all 13 patients showed increase in the \%BSI. Out of these 13 patients 12 were already in high risk group but 1 patients were in low risk group i.e. $\%$ BSI $<1$. When we compare results our findings with already published data [23-27]. Dennis et al in 88 patients showed that a doubling in BSI resulted in a 1.9-fold increase in risk of death. Log percent change in PSA at 6 months on treatment was also associated with survival in this study [28].

This data shows that \% BSI can predict outcome and in patients having initial high risk grade or positive change in grade (from lower to higher) carries poor prognosis. The Chi-squared statistic of log rank test is 6.232 with associated P-value $(0.013)$ of less than 0.05 rejects null hypothesis. The conclusion therefore is that, statistically, the two survival curves differ significantly, or that the grouping variable has a significant influence on survival time. Rejection of null hypothesis shows that two levels $<1$ and $>1$ are not identical regarding survival. The conclusion is that the curve representing the patients with the decrease tumour burden $(<1)$ has low risk and good survival then with the curve representing the patients $(>1)$ with more tumour burden.

For BLS 5 was taken as cut off. Below that patient were considered low risk and above that it was high risk. In Tables 4-6 it is shown that those patient whose BLS decreased as compared to the baseline scan showed better survival as compared to those who had increased in BLS on subsequent scan (7.6 \% vs. $92.3 \%)$. In Figures $4-12$ it is noticeable that in alive patients group most of the patients were showing decline in BLS $(\mathrm{N}=22)$. While in dead patients group all 12 out of 13 patients showed increase in the BLS. Out of these 13 patients 4 were already in high risk group but 7 patients were in low risk group i.e. BLS $<5$. The Chi-squared statistic of $\log$ rank test is 26.88 with associated P-value 0.000 less than 0.05 rejects null hypothesis. The conclusion therefore is that, statistically, the two survival curves differ significantly, or that the grouping variable has a significant influence on survival time. Rejection of null hypothesis shows that two levels $<5$ and $>5$ are not identical regarding survival. The conclusion is that the curve representing the patients with the decrease tumour burden $(<5)$ has good survival and decrease risk then with the curve representing the patients $(>5)$ with more tumour burden.

An overall trend seen in all serial scanning patients was that, there was decline in quantitative parameter numerical values or it remained stable in comparison with the patient which died where quantitative parameter numerical values were mostly increased. Although all parameters were able to predict survival and prognosis on change of parameter quantification results however, parameters which were based on number of lesions and not on involvement of skeletal percentage were not able to predict survival as accurately as others did. For example in EOD and in BLS the change of grade from 1 category to another was not that overt and many a time's patients were in the same group in which they were at baseline. Similarly when we see correlation with PSA, \%BSI and \%PAB performed better than the EOD and BLS. In regression analysis comparison with overall results $\mathrm{R}^{\wedge} 2$ were showing correlation but in t-statistics EOD was not correlating well with PSA. In survival analysis all parameters performed well and at give cut off point it was seen that low and high risk patient have marked difference in survival at 2 years.

So all quantitative parameters are strong predictors of tumour burden and are equally good in risk stratification too. The changes seen on serial bone scans reflected metastatic activity in the skeleton. Deterioration on the bone scan indicated disease progression or poor prognosis. Improvement on scan reflects regression of metastatic disease and usually implied a favorable survival. Consistent stabilization on the scan correlated with clinical stable disease and was associated with better survival than for the progressing patients.

\section{Conclusions}

All the four quantitative parameters (\% PAB, \%BSI, BLS and EOD) are good in quantifying the tumor burden and are good indicator in determining the disease status. $\% \mathrm{PAB}$ and \% BSI quantitative parameters are comparatively more accurate in calculating the tumor burden as compared to the EOD and BLS method. The disease prediction as progression or regression can easily be determined by using any of these four parameters.

Our present study suggests that \% $\mathrm{PAB}$ method is one of the 
Citation: Mustansar N (2018) Utility of Bone Scan Quantitative Parameters for the Evaluation of Prostate Cancer Patients. J Nucl Med Radiat Ther 9: 391. doi: 10.4172/2155-9619.1000391

Page 11 of 11

accurate methods in quantifying as a simple and reproducible estimate of the percentage of the skeleton involved in metastasis. It may also be very constructive to stratify patients in clinical trials. Large-scaled trials and further studies with more statistical power is required to assess the utility of serial \% PAB in monitoring the treatment effects and its worth as significant predictor of survival after hormonal treatment. \%BSI ontreatment changes are good response indicator and supports further exploration of bone scintigraphy to assess the treatment effects and survival prediction. The prostate cancer patients with the cut off \% BSI $>1, \% \mathrm{PAB}>0.5, \mathrm{BLS}>5$ and $\mathrm{EOD}$ with grade $2,3 \& 4$ showed disease progression and less survival.

\section{Limitations}

The limitation of our project is that we have included all the baseline bone scans of Carcinoma Prostate Patients irrespective of the treatment (Hormonal-Non Hormonal). Due to less time tenure of the project it was not possible to collect both the Baseline and the follow up scans of all the patients, though we have included 40 patients with both baseline and follow up scan (on hormonal treatment only) data. But in such small group of patients we cannot comment on the prognostic value and survival of the patients accurately.

The results are analyzed irrespective of the 'Flare Phenomenon' and that's why a lot of variation is observed.

\section{Reference}

1. Algra PRBJ, Tissing H, Falke TH, Arndt JW, Verboom LJ (1991) Detection of vertebral metastases: comparison between MR imaging and bone scintigraphy. Radiographics 11: 219-232.

2. Schneider A KL, Mattos AC, Keller ET, Allen MJ, Pienta KJ, et al. (2005) Bone turnover mediates preferential localization of prostate cancer in the skeleton. Endocrinology 146: 1727-1736.

3. Stroumbakis N, Cookson MS, Reuter VE, Fair WR (1997) Clinical significance of repeat sextant biopsies in prostate cancer patients. Oncology 49: 113-118.

4. Natarajan S, Marks LS, Margolis DJ, Huang J, Macairan ML, et al. (1997) Clinical application of a 3D ultrasound-guided prostate biopsy system. Uro Oncol 29: 334-342

5. Richmond CR (1985) International journal of radiation biology ICRP. Int $J$ Radiat Biol 48: 285

6. Cancer facts and figures (2007) American Cancer Society.

7. Sodeman TM, Batsakis JG (1977) Acid phosphatase in: Tannenbaum M, ed UrologicPathology: The Prostate. Philadelphia: Lea and Feibiger 2: 179-139.

8. Citrin DL, Cohen Al, Harberg J, Schlise S, Hougen C, et al. (1981) Systemic treatment of advanced prostatic cancer: Development of a new system for defining response. J Urol 125: 224-227.

9. Dennis ER, Jia X, Mezheritskiy IS, Stephenson RD, Schoder H, et al. Bone scan index: A quantitative a treatment response biomarker for castration resistant prostate cancer patients. J Clin Oncol 30: 519-524.

10. Noguchi M KH, Ishibashi M, Noda S. (2003) Percentage of the positive area of bone metastasis is an independent predictor of disease death in advanced prostate cancer. Br J Cancer 88: 195-201.

11. Soloway MS, Hardeman SW, Hickey D, Raymond J, Todd B, et al. (1988) Stratification of patients with metastatic prostate cancer based on extent of disease on initial bone scan. Cancer 61: 195-202.
12. Reza M , Bjartell A, Ohlsson M, Edenbrandt L, Tragardh E, et al. (2014) Increase in bone scan index during abiraterone treatment in relation to reduced survival in mCRPC patients. J Clin Oncol 4: 32.

13. Nakajima K, Nakajima $Y$, Horikoshi H, Ueno M, Wakabayashi $H$, et al. (2013) Enhanced diagnostic accuracy for quantitative bone scan using an artificial neural network system: A Japanese multi-center database project. EJNMMI Res 3: 83.

14. Tokuda O, Harada Y, Ohishi Y, Matsunaga N, Edenbrandt L (2014) Investigation of computer-aided diagnosis system for bone scans: A retrospective analysis in 406 patients. Annals of Nuclear Medicine 28: 329-339.

15. Pound CR, Brawer MK, Partin AW (2001) Evaluation and treatment of men with biochemical prostate-specific antigen recurrence following definitive therapy for clinically localized prostate cancer. Rev Urol 3: 72-84.

16. Tsai YS, Tzai TS, Johnny SN, Tong LYC, Yang WH (2005) Prognostic value of serial serum prostate-specific antigen measurements in metastatic prostate cancer patients treated with androgen ablation. JTUA 16: 65-72.

17. Noguchi M, Kikuchi H, Ishibashi M, Noda S (2003) Percentage of the positive area of bone metastasis is an independent predictor of disease death in advanced prostate cancer. Br J Cancer 88: 195-201.

18. Reza M, Bjartell A, Ohlsson M, Edenbrandt L, Tragardh E (2014) Increase in bone scan index during abiraterone treatment in relation to reduced survival in mCRPC patients. J Clin Oncol 4: 32.

19. Mizokami A, Izumi K, Ueno S, Kadono Y, Wakabayashi H (2014) Relationship of bone scan index and progression-free survival after docetaxel treatment for CRPC patients with bone metastases. J Clin Oncol 4:32.

20. Mitsui Y, Shiina H, Yamamoto Y, Haramoto M, Arichi N, et al. (2012) Prediction of survival benefit using an automated bone scan index in patients with castration-resistant prostate cancer. BJU Int 110: e628-e634.

21. Imbriaco M, Larson SM, Yeung HW, Mawlawi OR, Erdi Y, et al (1998) A new parameter for measuring metastatic bone involvement by prostate cancer: The Bone Scan Index. Clin Cancer Res 4: 1765-1772.

22. Noguchi M, Kikuchi H, Ishibashi M, Noda S (2003) Percentage of the positive area of bone metastasis is an independent predictor of disease death in advanced prostate cancer. Br J Cancer 88: 195-201.

23. Kaboteh R, Gjertsson P, Leek H, Lomsky M, Ohlsson M, et al. (2013) Progression of bone metastasis in prostate cancer patients- automated detection of new lesions and calculation of bone scan index. EJNMI research 3: 1-6.

24. Takahashi Y, Yoshimura M, Suzuki K, Hashimoto T, Hirose H, et al. (2012) Assessment of bone scans in advanced prostate carcinoma using fully automated and semi-automated bone scan index methods. Ann Nucl Med 26 : 586-593.

25. Reza (2012) Prognostic value of bone scan index for survival in patients with prostate cancer. EANM Annual Congress

26. Højgaard K (2010) Prognostic value of metastasis pattern for morbidity in prostate cancer measured by quantitative bone scintigraphy. ASCO Annual Meeting

27. Poulsen (2013) Bone Scan Index: A strong predictor of outcome in metastatic hormone native prostate cancer patients. SIU Congress

28. Dennis ER, Jia X, Mezheritskiy IS, Stephenson RD, Schoder H, et al. (2012) Bone scan index: A quantitative a treatment response biomarker for castration resistant prostate cancer patients. J Clin Oncol 30: 519-523. 